J. Austral. Math. Soc. 19 (Series A) (1975), 238-246.

\title{
FINITELY GENERATED PSEUDOCOMPLEMENTED DISTRIBUTIVE LATTICES
}

J. BERMAN and PH. DWINGER

(Received 22 November 1973)

Communicated by B. Mond

If $L$ is a pseudocomplemented distributive lattice which is generated by a finite set $X$, then we will show that there exists a subset $G$ of $L$ which is associated with $X$ in a natural way such that $|G| \leqq|X|+2^{|X|}$ and whose structure as a partially ordered set characterizes the structure of $L$ to a great extent. We first prove in Section 2 as a basic fact that each element of $L$ can be obtained by forming sums (joins) and products (meets) of elements of $G$ only. Thus, $L$ considered as a distributive lattice with 0,1 (the operation of pseudocomplementation deleted), is generated by $G$. We apply this to characterize for example, the maximal homomorphic images of $L$ in each of the equational subclasses of the class $B_{\omega}$ of pseudocomplemented distributive lattices, and also to find the conditions which have to be satisfied by $G$ in order that $X$ freely generates $L$.

In Section 3 we investigate the pseudocomplemented meet semilattice $\bar{G}$ which is generated by $G$ for the case that $L$ is freely generated by $X$. It is shown that $\bar{G} \sim\{0\}$ is exactly the set of join-irreducibles of $L$ (Urquhart (to appear)). Furthermore we show that $\bar{G}$ is the pseudocomplemented meet-semilattice which is freely generated by $X$ (cf. Balbes (1973)) and that $L$ is isomorphic to the algebra freely generated by $\bar{G}$ over the class of distributive lattices, where $\bar{G}$ is considered as a partial lattice.

It follows from the basic result in Section 2 mentioned above, that $L$ considered as a distributive lattice with 0,1 , is a lattice homomorphic image of the distributive lattice with 0,1 which is freely generated by a set of cardinality $|G|$. It is a natural question to ask whether $|G|$ is minimal with this property. This question is answered in Section 4 in the affirmative.

In Section 5 we generalize some of the results obtained in the previous sections to the case that $L$ is infinite.

\section{Preliminaries}

For the notions of algebra, subalgebra, partial algebra, relative (partial) 
algebra, homomorphism between partial algebras, principal congruence relation, maximal homomorphic image, etc. we refer the reader to Grätzer (1968). We will often denote a (partial) algebra $\langle A, F\rangle$ by the symbol $A$ only. If $A$ and $B$ are (partial) algebras of the same similarity type then $[A, B]$ will denote the set of homomorphisms from $A$ to $B$. It will often be useful, if we deal with a class $V$ of (partial) algebras of a certain similarity type and if $A$ and $B \in V$, to write $[A, B]_{V}$ instead of $[A, B]$. If $V$ is an equational class of algebras and $A \in V, T \subseteq A$, then $[T]_{V}$ will denote the subalgebra of $A$ generated by $T$. If $T=\left\{x_{1}, \cdots, x_{n}\right\}$, then we will write $\left[x_{1}, \cdots, x_{n}\right]_{V}$ instead of $\left[\left\{x_{1}, \cdots, x_{n}\right\}\right]_{V}$. If $V$ is an equational class of algebras then $F V(X)$ denotes the free algebra over $V$ on a free generating set $X$. If $|X|=\alpha$, then we also use the symbol $F V(\alpha)$. Again, if $V$ is an equational class and $A$ is a partial algebra of the same similarity type then $F V(A)$ denotes the algebra freely generated by $A$ over $V$. Thus $F V(A) \in V$ and there exists an isomorphism $f$ between $A$ and a relative subalgebra $A^{\prime}$ of $F V(A)$ such that $\left[A^{\prime}\right]_{V}=F V(A)$ and for each $g \in[A, B]$, there exists an $h \in[F V(A), B]$ with $h \cdot f=g$.

Of particular interest in this paper are the equational classes of algebras:

$D$ : distributive lattices with operations $\cdot$ and + .

$D_{01}$ : distributive lattices with 0,1 and operations $+, \cdot, 0,1$.

$B_{\omega}$ : pseudocomplemented distributive lattices with operations $+, \cdot, *, 0$.

$M$ : pseudocomplemented (meet) semilattices with operations $\cdot,{ }^{*}, 0$.

The operation $*$ in $B_{\omega}$ and $M$ is defined by $x x^{*}=0$ and if $x y=0$, then $y \leqq x^{*}$. For the properties of these classes see Grätzer (1968), Frink (1962) and Balbes (1973) Recall that for $L \in B_{\omega}$ or $L \in M$ we have for $x, y \in L$.

1.1 (i) $x \leqq y$ implies $x^{*} \geqq y^{*}$

(ii) $x \leqq x^{* *}$

(iii) $x^{*}=x^{* * *}$

The two element Boolean algebra is denoted by 2 and $2^{m} \oplus 1, m \geqq 0$ stands for the algebra obtained from $2^{m}$ by adjoining another one element. Note $2^{m} \oplus 1 \in B_{\omega}$ for $m \geqq 0$. For $L \in B_{\omega}$, we let $S(L)=\left\{x^{*} \mid x \in L\right\}$. It is well known that $S(L)$ is a Boolean algebra under the partial ordering of $L$. It is known that besides $B_{\omega}$ the only equational subclasess of $B_{\omega}$ are the classes $B_{m}, m=-1,0,1, \cdots$ and where $B_{-1}$ is the trivial class and where for $m \geqq 0 B_{m}$ is the class generated by $2^{m} \oplus 1$ (Lakser (1971), Lee(1970)). If $L \in B_{\omega}$, then $L \in B_{\omega}, m \geqq 1$, is equivalent to either of the following conditions (Grätzer (1971)).

1.2. For $z_{0}, z_{1}, \cdots, z_{m} \in L$ :

(i) $\left(z_{1} z_{2} \cdots z_{m}\right)^{*}+\left(z_{1}^{*} z_{2} \cdots z_{m}\right)^{*}+\cdots+\left(z_{1} z_{2} \cdots z_{m}^{*}\right)^{*}=1$

(ii) if $z_{i} z_{j}=0$ for all $i \neq j$, then $z_{v}^{*}+z_{1}^{*}+\cdots+z_{m}^{*}=1$.

Finally, for notational convenience, if $X$ is a set, $T \subseteq X$ means $T$ is a finite non-void subset of $X$. 


\section{Lattice theoretic generation of $B_{\omega}$ algebras}

It is well known that the congruence relation $\sim$ on $L \in B_{\omega}$ defined by $x \sim y$ if and only if $x^{*}=y^{*}$ is such that $S(L) \cong L / \sim$. In the following lemma we give an alternate characterization of this congruence relation for the case that $L$ is finite. This result will be used to characterize the ${ }^{*}$ operation of $L, L$ finite, in terms of the atoms of $L$.

2.1 Lemma. Let $L \in B_{\omega}, L$ finite. For $x, y \in L$, define $x \equiv y$ if and only if $\{a \in L \mid a$ is an atom of $L, a \leqq x\}=\{a \in L \mid a$ is an atom of $L, a \leqq y\}$. Then $x \equiv y$ if and only if $x^{*}=y^{*}$.

Proof. Let $x \in L$. Let $y=\Sigma\{a \in L \mid a$ is an atom, $a \neq x\}$. Let $z=\Sigma\{w \in L \mid$ $w \equiv y\}$. Then by distributivity $z \equiv y$. Hence $x z=0$. Moreover, suppose $x u=0$ for some $u \in L$. It must be that $\{a \in L \mid a$ is an atom, $a \leqq u\} \subseteq\{a \in L \mid a$ is an atom, $a \leqq y\}$. So $u \leqq z$. Hence $z=x^{*}$. The lemma now follows.

2.2 Notation. Let $L \in B_{\omega}$ with $L=\left[x_{1}, \cdots, x_{n}\right]_{B_{\omega}}$. Define $x_{i}^{0}=x_{i}$ and $x_{i}^{1}=x_{i}^{*}$. For $1 \leqq j \leqq 2^{n}$, let $a_{j}=x_{1}^{\varepsilon_{1}} \cdots x_{n}^{\varepsilon_{n}}$, with $\left(\varepsilon_{1}, \cdots, \varepsilon_{n}\right) \in\{0,1\}^{n}$. Define $b_{j}=a_{j}^{*}$. For $1 \leqq i \leqq n$ and $1 \leqq j \leqq 2^{n}$ let $\left(a_{j}\right)_{i}=\left(b_{j}\right)_{i}=\varepsilon_{i}$. Also let $X=\left\{x_{1}, \cdots, x_{n}\right\}, A=\left\{a_{1}, \cdots, a_{2^{n}}\right\}, B=\left\{b_{1}, \cdots, b_{2^{n}}\right\}$ and $G=X \cup B$. In the sequel, the sets $G$ and $B$ will be of particular interest. In this section we will show that the partial order structure of $G$ and $B$ determine the algebraic structure of $L$.

2.3 Lemma. Let $L \in B_{\omega}$ with $L=\left[x_{1}, \cdots, x_{n}\right]_{B_{\omega}}$. Then each $a_{i} \in A$ is an atom or 0 . Moreover, every atom in $L$ is equal to some $a_{i}$ for exactly one $i$.

Proof. Clearly $x_{j} \cdot a_{i} \in\left\{0, a_{i}\right\}$ for all $x_{j}$. Let $y, z \in L$ be such that $y a_{i} \in\left\{0, a_{i}\right\}$ and $z a_{i} \in\left\{0, a_{i}\right\}$. Certainly $(y z) a_{i} \in\left\{0, a_{i}\right\}$ and by distributivity $(y+z) a_{i} \in\left\{0, a_{i}\right\}$. If $y a_{i}=0$, then $a_{i} \leqq y^{*}$. Thus $y^{*} a_{i}=a_{i}$ If $y a_{i}=a_{i}$, then $y \geqq a_{i}$. So by 1.1 , $y^{*} \leqq a_{i}^{*}$, so $y^{*} a_{i}=0$. Since $L=\left[x_{1}, \cdots, x_{n}\right]_{B_{\omega}}$ this completes the proof of the first claim. Next observe that $s=\Sigma\left\{a_{i} \mid 1 \leqq i \leqq 2^{n}\right\}=\left(x_{1}+x_{1}^{*}\right) \cdots\left(x_{n}+x_{n}^{*}\right)$. Hence $s^{*}=1^{*}$, so by 2.1 every atom is equal to some $a_{i}$. If $a_{i}=a_{j}$ in $L$, for $i \neq j$, then there exists $k$ for which $\left(a_{i}\right)_{k} \neq\left(a_{j}\right)_{k}$. So $a_{i} \leqq x_{k} x_{k}^{*}=0$.

From 2.1 it follows that if $a$ is an atom of $L$, then $a^{*}$ is a dual atom of $S(L)$. So from 2.3 it follows that each $b_{i} \in B$ is either 1 or a dual atom in the Boolean algebra $S(L)$. Moreover, every dual atom of $S(L)$ is equal to exactly one $b_{i}$. Thus, $S(L)$ is generated by $B$ under the formation of products. (Note $\Pi \phi=1$ ). Indeed, let $z^{*} \in S(L)$. Form $T=\left\{b_{i} \mid a_{i} \leqq z\right\}$. It is easily seen that $z^{*}=\Pi T$.

2.4 TheOREM. Let $L \in B_{\omega}, L=[X]_{B_{\omega}}, X$ finite. Then $L=[G]_{D_{01}}$.

Proof. Since $L=[X]_{B_{\omega}}$ and $X \subseteq G$, only applications of * need be considered. By the remarks following 2.3 , any application of $*$ is equivalent to 
forming $\Pi T$ for some $T \subseteq B \subseteq G$. Indeed, if $z \in L$, then $z=\Pi T_{1}+\cdots+\Pi T_{r}$ for some family of sets $T_{i} \subseteq G, 1 \leqq i \leqq r$.

2.6 THEOREM. Let $L \in B_{\omega}, L=\left[x_{1}, \cdots, x_{n}\right]_{B_{\omega}}$. Then $L \in B_{\omega}, m \geqq 1$, if and only if for all $I \subseteq\left\{1,2, \cdots, 2^{n}\right\}$ such that $|I| \geqq m+1$, the equality $\sum_{i \in I} b_{i}=1$ holds.

Proof. By 2.3, $a_{i} a_{j}=0$ for $i \neq j$. So by $1.2 \sum_{i \in I} b_{i}=1$. Conversely, suppose $\left\{y_{0}, y_{1}, \cdots, y_{m}\right\} \subseteq L$ and $y_{i} y_{j}=0$ for $i \neq j$. Note $y_{i}^{*}=\Pi\left\{b_{k} \mid a_{k} \leqq y_{i}\right\}=\Pi T_{i}$. Hence $T_{i} \cap T_{j}=\varnothing$ or $\{1\}$. So $\Sigma_{i=0}^{m} y_{i}^{*}=\Sigma_{i=0}^{m}\left(\Pi T_{i}\right)=\left(\Sigma Q_{1}\right) \cdots\left(\Sigma Q_{r}\right)$ where each $Q_{i}$ contains the element 1 or $m+1 b_{j}$. Hence $\Sigma Q_{i}=1$ for all $i$.

2.7 Theorem. Let $L=\left[x_{1}, \cdots, x_{n}\right]_{B_{\omega} \cdot}$ Define $u_{m}=\Pi\{\Sigma S|S \subseteq B| S \mid$, $=m+1\}$. Let $\theta\left(u_{m}, 1\right)$ be the principal $B_{\omega}$ congruence relation generated by $\left\{u_{m}, 1\right\}$. Define $L_{m}=L / \theta\left(u_{m}, 1\right)$. Then all of the following hold:

i) $L_{m} \in B_{m}$

ii) $L_{m}$ is a maximal homomorphic image of $L$ in $B_{m}$

iii) $L_{m}$ is isomorphic to the interval $\left[0, u_{m}\right] \subseteq L$.

Proof. By 2.6, $L_{m} \in B_{m}$. If $L / \theta=L_{1} \in B_{m}$ then again by $2.61 \equiv u_{m}(\theta)$. Hence, $\theta \geqq \theta\left(u_{m}, 1\right)$, so (ii) holds. Observe that since $\theta\left(u_{m}, 1\right)$ is determined by a principal filter, $x \equiv y\left(\theta\left(u_{m}, 1\right)\right)$ if and only if $x u_{m}=y u_{m}$ (Lakser (1973)). So every congruence class of $L_{m}$ contains exactly one element in [0, $\left.u_{m}\right]$. Hence (iii) follows.

We now specialize to the case where $L$ is free in $B_{\omega}$.

2.8 TheOREM. Let $\left.G=X \cup B \subseteq F B_{\omega}{ }^{\prime} X\right)$ with $X=\left\{x_{1}, \cdots, x_{n}\right\}$. For $S, T \subseteq G, \Pi S \leqq \Sigma T$ if and only if at least one of the following hold:

(i) $S \cap T \neq \varnothing$.

(ii) There exist $1 \leqq j \leqq 2^{n}$ and $1 \leqq i \leqq n$ with $b_{j} \in T$ and $\left(b_{j}\right)_{i}=1$ for some $x_{i} \in S$.

(iii) $B=\left\{b_{j} \mid b_{j} \in S\right\} \cup\left\{b_{j} \mid\left(b_{j}\right)_{i}=1, x_{i} \in S\right\}$.

For $F B_{m}(X)$ the following condition may be added to the list:

(iv) $|T \cap B|>m$.

Proof. $\leftarrow$ (i) suffices in any lattice. For (ii) observe that $\left(b_{j}\right)_{i}=1$ implies $x_{i}^{*} \geqq a_{j}$. So by $1.1 x_{i} \leqq x_{i}^{* *} \leqq b_{j} \in T$. If (iii) holds then $\Pi S \leqq \Pi B=0$. In the case of $F B_{m}(X)$, if $|T \cap B|>m$, then $\Sigma T=1$ by 2.6. $\Rightarrow$ Suppose in $F B_{\omega}(X)$ $\Pi S \leqq \Sigma T$ and neither (i), (ii) nor (iii) hold. Note that since the Boolean algebra $2^{2^{n}}$ is a $B_{\omega}$ homomorphic image of $F B_{\omega}(n)$, each of the $b_{i}, 1 \leqq i \leqq 2^{n}$, are distinct in $F B_{\omega}(n)$. Let $|T \cap B|=t$. If $t=0$, by the negation of (iii) $a b_{j}$ may be adjoined to $T$ for which conditions (i) (ii) nor (iii) will still not hold. So assume $|T \cap B|$ $=t \geqq 1$. There exists $f \in\left[F B_{\omega}(X), 2^{t}\right]_{B \ldots}$ such that $f\left(a_{i}\right)$ is an atom of $2^{t}$ for all $i$, $b_{i} \in T, f\left(a_{i}\right)=0$ otherwise. Adjoin a new maximal element $1^{\prime}$ to $2^{t}$ to obtain $L=2^{t} \oplus 1^{\prime}$. Thus $L \in B_{\omega}, 0^{*}=1^{\prime}$ and $1^{\prime}$ is join-irreducible. Assume $x_{i} \in S$ for 
$1 \leqq i \leqq k, x_{i} \notin S, i>k$. Define $\gamma: X \rightarrow L$ by $\gamma\left(x_{i}\right)=1^{\prime}$ for $1 \leqq i \leqq k, \gamma\left(x_{i}\right)$ $=f\left(x_{i}\right)$ otherwise. Let $g \in\left[F B_{\omega}(n), L\right]_{B_{w}}$ extend $\gamma$. If $x_{i} \in T$, then by the negation of (i), $g\left(x_{i}\right)<1^{\prime}$. If $b_{j} \in T$, then by the negation of (ii) $g\left(a_{j}\right) \geqq f\left(a_{j}\right)$ so $1^{\prime}>f\left(b_{j}\right)$ $\geqq g\left(b_{j}\right)$. Thus $g(\Sigma T)<1^{\prime}$. If $x_{i} \in S$ or $b_{j} \in S$ with $\left(b_{j}\right)_{i}=1$ for some $1 \leqq i \leqq k$, then $g\left(x_{i}\right)=g\left(b_{j}\right)=1^{\prime}$. So suppose $b_{j} \in S, a_{j}=x_{1} \cdots x_{k} x_{k+1}^{\varepsilon_{k}+1} \cdots x_{n}^{\varepsilon_{n}}$. Let $C=\left\{a_{i} \mid\left(a_{j}\right)_{r}=\left(a_{i}\right)_{r}\right.$ for all $\left.r>k\right\}$. Then $0=\Sigma f(C)=f\left(x_{k+1}^{\varepsilon k+1} \cdots x_{n}^{\varepsilon_{n}}\right)$ $g\left(x_{k+1}^{\varepsilon_{k+1}} \cdots x_{n}^{c_{n}}\right)$. Thus $g\left(b_{j}\right)=1^{\prime}$ also. So $g(\Pi S)=1^{\prime}$. This contradicts the assumption $\Pi S \leqq \Sigma T$. For the case of $F B_{m}(X)$, note that if (iv) does not hold, then $t=|T \cap B| \leqq m$. But $\mathbf{2}^{t} \oplus \mathbf{1}^{\prime} \in B_{m}$ for $t \leqq m$. So the above contradiction can be obtained.

2.9 THEOREM. The lattice $F B_{\omega}(n)$ contains for each $m<\omega$ an ideal which is lattice isomorphic to $F B_{m}(n)$. Moreover, these ideals form a chain when ordered by inclusion.

Proof. Since $B_{m} \subseteq B_{\omega}, F B_{m}(n)$ is a homomorphic image of $F B_{\omega}(n)$. Let $\theta_{m}$ be a congruence on $F B_{\omega}(n)$ such that $F B_{\omega}(n) / \theta \simeq F B_{m}(n)$. Apply 2.7 to show $\theta_{m}=\theta\left(u_{m}, 1\right)$. So by 2.7 (iii) the ideal $\left[0, u_{m}\right]$ is lattice isomorphic to $F B_{m}(n)$. Finally, note $u_{1} \leqq u_{2} \leqq \cdots u_{m} \leqq \cdots$ in $F B_{\omega}(n)$.

Next, independence conditions are obtained for $B_{\omega}$ and $B_{m}$. See Marczewski (1958) of Grätzer (1968) for a general discussion of independence.

2.10 THEOREM. Let $L \in B_{\omega}, L=\left[x_{1}, \cdots, x_{n}\right]_{B_{\omega}} . L \simeq F B_{\omega}(n)$ if and only if whenever $S, T \subseteq$. $G$ and $\Pi S \leqq \Sigma T$, then one or more of 2.8 (i), (ii) or (iii) hold. Moreover, if $L \in B_{m}$, then $L \simeq F B_{m}(n)$ if and only if the additional condition of 2.8 (iv) is included.

Proof. $\Rightarrow$ Use 2.8. $\Leftarrow$ Let $X=\left\{x_{1}, \cdots, x_{n}\right\}$. Define $\gamma: X \rightarrow L$ by $\gamma\left(x_{i}\right)=x_{i}$. Then $\gamma$ extends to a $B_{\omega}$ homomorphism $g$ from $F B_{\omega}(n)\left(F B_{m}(n)\right)$ onto $L \in B_{\omega}$ ( $L \in B_{m}$ ). A standard argument shows that conditions (i) (ii) (iii) (and (iv)) guarantee that $g$ is also one-to-one.

2.11 CoRollaRY (Grätzer, Lakser (1971; page 190)), For $k \geqq 2^{n}, F B_{\omega}(n)$ $\simeq F B_{k}(n)$.

Proof. For $k \geqq 2^{n}$, since $|B| \leqq 2^{n}$, condition 2.8 (iv) cannot hold.

\section{The semilattice generated by $G$}

3.1 Notation. Consider $L \in B_{\omega}, L=[X]_{B_{\omega}}$ with $X$ finite. Let $G$ be as in 2.2. Form $\bar{G}=\{\Pi T \mid T \subseteq G\}$. Thus, $\bar{G}$ is the closure of $G$ under the formation of products. Observe $\Pi \phi=1 \in \bar{G}$ and from the remarks following $2.3, S(L) \subseteq \bar{G}$. Also, 2.4 implies that the set of join-irreducible elements of $L$ is contained in $\bar{G}$.

3.2 Notation. Let $\bar{G}$ be as in 3.1 with $z \in \bar{G}$. Let $\beta(z)=\left\{b_{i} \in B \mid b_{i} \geqq z\right\}$ and $\chi(z)=\left\{x_{i} \in X \mid x_{i} \geqq z\right\}$. Then $z=(\Pi \beta(z))(\Pi \chi(z))$. 
In the remainder of this section we will be concerned with the set $\bar{G} \subseteq F B_{\omega}(X)$ or $G \subseteq F B_{m}(X)$ for $|X|=n, n$ finite.

3.3 THEOREM. The join-irreducible elements of $F B_{m}(n)$ are precisely those $z \in \bar{G}, z \neq 0$, for which $2^{n}>|\beta(z)| \geqq 2^{n}-m$. In particular, the set of join-irreducibles of $F B_{\omega}(X)$ is $\bar{G} \backslash\{0\}$.

Proof. If $z \in \bar{G}$ and if $|\beta(z)|=2^{n}$, then $z=0$. If $|\beta(z)|<2^{n}-m$, then there exist, say $b_{0}, \cdots, b_{m} \in B$, with $b_{i} \geq z$ for $0 \leqq i \leqq m$. Thus in $F B_{m}(n)$ we have $z=z \cdot 1=z\left(b_{0}+\cdots+b_{m}\right)=z b_{0}+\cdots+z b_{m}$. Finally, suppose $2^{n}>|\beta(z)| \geqq 2^{n}-m$. Let $z=\Pi T_{1}+\cdots+\Pi T_{r}$, with $T_{i} \subseteq G$. If $\Pi T_{i} \neq z$ for all $i$, then for each $i$ there exist $t_{i} \in T_{i}$ such that $t_{i} \geq$.

Thus

$$
0 \neq z=(\Pi \beta(z))(\Pi \chi(z)) \leqq t_{1}+\cdots+t_{r} .
$$

Since $\left|\left\{t_{1}, \cdots, t_{r}\right\} \cap B\right| \leqq m, 2.8$ gives a contradiction. So $z$ is join-irreducible. The second part of the theorem follows from 2.11.

For an alternate characterization of the join-irreducibles of $F B_{m}(n)$ and $F B_{\omega}(n)$ see Urquhart (to appear).

We now determine the number of join-irreducible elements in $F B_{m}(n)$ and $F B_{\omega}(n)$. This, of course, gives the lengths of these lattices. Compare Balbes (1973).

3.4 THEOREM. Define $p(s, t)=\sum_{i=1}^{i}\left(\begin{array}{c}2 s \\ i\end{array}\right)$. Then the number of joinirreducible elements of $F B_{m}(n)$ is $\sum_{k=0}^{n}\left(\begin{array}{c}n \\ k\end{array}\right) p(n-k, m)$. In particular, for $F B_{\omega}(n)$ this is equal to $\sum_{k=0}^{n}\left(\begin{array}{l}n \\ k\end{array}\right)\left(2^{2^{k}}-1\right)$.

PROOF. If $z=(\Pi \beta(z))(\Pi \chi(z))$ with $|\chi(z)|=k$, then $B \backslash \beta(z)$ is contained in a set of cardinality $2^{n-k}$. Since $B \backslash \beta(z) \neq \varnothing$, there are $p(n-k, m)$ choices for $\beta(z)$. Hence, $\left(\begin{array}{l}n \\ k\end{array}\right) p(n-k, m)$ possible choices for $z$. Finally observe that if $m \geqq 2^{n}$, then $p(n-k, m)=2^{2^{k}}-1$ for all $k$. Apply 2.11 to complete the proof.

3.5 ReMARK. Note that $\bar{G}$ is closed with respect to $\cdot$ and *. If $\bar{G}$ is considered as a relative partial $B_{\omega}$ subalgebra of $F B_{\omega}(n)$, then for $y, z \in \bar{G}, y+z$ is defined if and only if $y+z \in \bar{G}$. So by 3.3,y+z is defined if and only if $y$ and $z$ are comparable elements of $F B_{\omega}(n)$.

3.6 THEOREM. Let $\bar{G} \subseteq F B_{\omega}(X),|X|$ finite, be as in 3.1. Consider $\bar{G}$ as a partial lattice where $x+y$ is defined if and only if $x$ and $y$ are comparable. Then $F B_{\omega}(X)$ is the distributive lattice freely generated over $D$ by the partial lattice $\bar{G}$.

Proof. By $3.5 G$ is a relative partial lattice and by $2.4[\bar{G}]_{D}=F B_{\omega}(X)$. Let $L \in D$ and $f \in[\bar{G}, L]_{D}$. Since $L_{1}=[f(\bar{G})]_{D}$ is finite, $L_{1} \in B_{\omega}$. So the function $f$ restricted to $X$ has an extension to $g \in\left[F B_{\omega}(\bar{X}), L_{1}\right]_{B_{\omega}}$. But since $G \subseteq[X]_{D}$ and $f$ is a partial homomorphism, $g$ extends $f$ as well. So $g \in\left[F B_{\omega}(X), L\right]_{D}$. 
The next lemma gives an embedding of an arbitrary pseudocomplemented semilattice in a pseudocomplemented lattice. See also Balbes (1969) and Dyson (1965).

3.7 Lemma. Let $S \in M, S$ arbitrary. There exists $L \in B_{\omega}$ such that the reduct $L^{\prime}=\langle L, \cdot, *, 0\rangle$ of $L$ has a subalgebra $S^{\prime}$ which is $M$-isomorphic to $S$. Moreover, $\left[S^{\prime}\right]_{D}=L$.

Proof. For $a \in S$ let $(a]=\{z \in S \mid 0 \leqq z \leqq a\}$. Form $S^{\prime}=\{(a] \mid a \in S\}$. Let $L$ be the ring of sets generated by $S^{\prime}$. It is easily verified that $S^{\prime}$ is a pseudocomplemented semilattice with zero element $(0]$, with $\cap$ for product and with $(a]^{*}=\left(a^{*}\right]$. To complete the proof it remains to show $L \in B_{\omega}$. Let $T \in L$. So there exist $t_{1}, \cdots, t_{p} \in S$ such that $T=\left(t_{1}\right] \cup \ldots \cup\left(t_{p}\right]$. Omit all $t_{i}$ for which $t_{i} \leqq t_{j}$ for some $j \neq i$. Then this is a unique representation for $T$. For, if otherwise, $T=\left(t_{1}\right] \cup \cdots \cup\left(t_{p}\right]=\left(r_{1}\right] \cup \cdots \cup\left(r_{q}\right]$. So for any $i, t_{i} \leqq r_{j}$ for some $j$. Similarly $r_{j} \leqq t_{k}$. Thus $t_{i} \leqq t_{k}$ so $t_{i}=r_{j}=t_{k}$. Thus $\left\{t_{1}, \cdots, t_{p}\right\}=\left\{r_{1}, \cdots, r_{q}\right\}$. Define $T^{*}=\left(t_{1}^{*}\right] \cup \ldots \cup\left(t_{p}^{*}\right]$. By the above, $T^{*}$ is well defined and easily seen to be the pseudocomplement of $T$ in $L$.

3.8 Corollary. Let $S \in M$ be an arbitrary pseudocomplemented semilattice. Consider $S$ as a partial $B_{\omega}$ lattice where $x+y$ is defined if and only if $x$ and $y$ are comparable. Then the $B_{\omega}$ lattice $L$ constructed from $S$ in 3.7 is isomorphic to the $B_{\omega}$ lattice freely generated by the partial $B_{\omega}$ algebra $S$.

Proof. It is easily seen that the set $S^{\prime} \backslash(0]$ consists only of join-irreducibles in $L$. So $S^{\prime}$ is a relative partial $B_{\omega}$ subalgebra of $L$. Using the uniqueness of the representation of elements of $L$ as union of principal ideals in $S$, the mapping extension property can be verified.

3.9 THEOREM. Let $\bar{G} \subseteq F B_{\omega}(n)$, $n$ finite. Then $\bar{G}$ is $M$-isomorphic to $F M(n)$.

Proof. Let $X=\left\{x_{1}, \cdots, x_{n}\right\}$ and $Y=\left\{y_{1}, \cdots, y_{n}\right\}$. Consider arbitrary $S \in M, S=[Y]_{M}$. Suppose $F B_{\omega}(n)=[X]_{B_{\omega}}$. Construct $S^{\prime}$ and $L$ as in 3.7. Identify $S$ with $S^{\prime} \subseteq L$. Observe $[Y]_{B_{\omega}}=L$. Let $\gamma\left(x_{i}\right)=y_{i}$ and extend $\gamma$ to $g \in\left[F B_{\omega}(n), L\right]_{B_{\omega}}$. Then $g(\bar{G})$ is closed under $\cdot$ and $*$ and contains 0 and $Y$. Hence $S=g(\bar{G})$. So $g \in[\bar{G}, S]_{M}$ and $g$ extends $\gamma$ as desired.

For an alternate characterization of $F M(n)$, see Balbes (1973).

\section{Minimality}

It follows from 2.4 that $F B_{\omega}(n)$ is a $D_{01}$ homomorphic image of $F D_{01}\left(n+2^{n}\right)$. A natural question is whether the number $n+2^{n}$ is minimal with this property. Or equivalently, does there exist a subset $S \subseteq F B_{\omega}(n),|S|<n+2^{n}$, for which $F B_{\omega}(n)=[S]_{D_{01}}$ ? 
4.1 LEMMA. Every element of $B \subseteq F B_{\omega}(n)$ is both meet-irreducible and join-irreducible.

Proof. Let $b_{j} \in B$. By $2.8 b_{j}$ is join-irreducible. Suppose $b_{j}=p q$. By 2.4 we may write $p=\left(\Sigma S_{1}\right) \cdots\left(\Sigma S_{k}\right)$ and $q=\left(\Sigma T_{1}\right) \cdots\left(\Sigma T_{l}\right)$, with $S_{i}, T_{i} \subseteq G$. Use 2.8 and an argument similar to that in 3.3 to show that for some $i, b_{j} \geqq \Sigma S_{i}$ or $b_{j} \geqq \Sigma T_{i}$.

4.2 THEOREM. If $F B_{\omega}(n)=[Y]_{D_{01}}$, then $|Y| \geqq n+2^{n}$.

Proof. By $4.1 B \subseteq Y$. Each $x_{i}, 1 \leqq i \leqq n$, in the free generating set for $F B_{\omega}(n)$ is join-irreducible. So $x_{i}=\Pi S_{i}, S_{i} \subseteq Y$. Define $T_{i}=\left\{y \in Y \mid y \in S_{i}, y \notin B\right\}$. By $2.8, T_{i} \neq \varnothing$ for each $i$. Let $b_{1} \in B$ be such that $\left(b_{1}\right)_{i}=0$ for all $i, 1 \leqq i \leqq n$. If for some $i, T_{i} \subseteq \cap_{j \neq i} T_{j}$, then $S_{i} \subseteq\left(\cup_{j \neq i} T_{j}\right) \cup\left(B \backslash\left\{b_{1}\right\}\right)$. Hence $x_{i}$ $\geqq\left(\Pi_{j \neq i} x_{j}\right)\left(\Pi\left(B \backslash\left\{b_{1}\right\}\right)\right)$. This violates 2.8. So each $T_{i}$ contains, say, $y_{i}$ for which $y_{i} \notin B$ and $y_{i} \notin T_{j}$ for $j \neq i$. Thus $|Y| \geqq n+2^{n}$.

It is interesting to note that 4.2 is not true for $F B_{m}(n), m$ arbitrary.

\section{The infinite case}

In this final section we generalize some of the results of the previous sections to $F B_{\omega}(X)$, where $X$ is an infinite set of arbitrary cardinality.

5.1 Definition. For $Y \subseteq \cdot X$, define $B(Y) \subseteq F B_{\omega}(X)$ by

$$
B(Y)=\left\{\left[\left(\Pi S^{*}\right)(\Pi((Y \backslash S))]^{*} \mid S \subseteq Y\right\} .\right.
$$

Let $B=\cup\{B(Y) \mid Y \subseteq \cdot X\}$. Form $G=X \cup B$.

5.2 THEOREM. $F B_{\omega}(X)=[G]_{D_{01}}$. Moreover, if $\alpha$ is any infinite cardinal, $F B_{\omega}(\alpha)$ is a $D_{01}$ homomorphic image of $F D_{01}(\alpha)$.

Proof. For $z \in F B_{\omega}(X), z$ may be obtained from a finite subset $Y \subseteq X$ by a finite series of applications of,$+ \cdot$ and *. Apply 2.4 to show $[Y]_{B_{c},}$

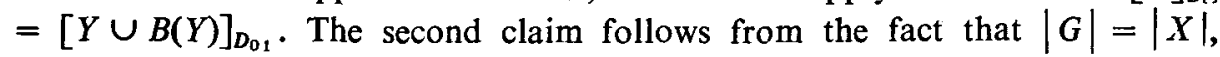
whenever $X$ is infinite.

5.3 Remark. If $z_{1}, \cdots, z_{k} \in F B_{\omega}(X)$, then there exists some set $Y \subseteq X$ such that $z_{1}, \cdots, z_{k} \in[Y]_{B_{\omega}}$. Observe $[Y]_{B_{\omega,}} \cong F B_{\omega}(Y)$. Thus, the results of sections 2 and 3 apply to $z_{1}, \cdots, z_{k}$. In particular, for $X$ infinite and $G$ as in 5.1, define $G=\{\Pi T \mid T \subseteq G, T$ finite $\}$. It can be seen that the set of join-irreducible

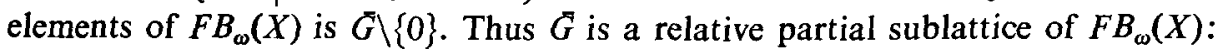
$y+z$ is defined only in the case that $y$ and $z$ are comparable. Arguments similar to those in Section 3 give the following:

5.4 THEOREM. $F B_{\omega}(X)$ is the distributive lattice freely generated in $D$ by the partial lattice $G$. 
5.5 THEOREM. The pseudocomplemented semilattice $\bar{G}$ is $M$ isomorphic to $F M(X)$.

Recall that for $L \in D$, a subset $F \subseteq L$ is a prime filter if and only if there exists $h \in[L,\{0,1\}]_{D}, h$ onto such that $h^{-1}(1)=F$. Observe that every proper filter in the partial lattice $\bar{G}$ is a prime. Apply 5.4 to obtain

5.6 THEOREM. The partially ordered set of prime filters of $F B_{\omega}(X)$ is isomorphic to the partially ordered set of proper filters of $\bar{G}$.

\section{References}

R. Balbes (1969), 'A representation theory for prime and implicativde semilattices', Trans. Amer. Math. Soc. 136, 261-267.

R. Balbes (1973), 'On free pseudo-c'omplemented and relatively pseudo-complemented semilattices', Fund. Math. 8, 119-131.

V. Dyson (1965), An embedding theorem for semilattices, (Hughes Aircraft Technical Report, 1965).

O. Frink (1962), 'Pseudo-complements in semi-lattices', Duke Math. J. 29, 505-514.

G. Grätzer (1968), Universal Algebra, (Van Nostrand, 1968).

G. Grätzer (1971), Lattice Theory, (W. H. Freeman and Co., 1971).

H. Lakser (1971), 'The structure of pseudo-complemented, distributive lattices I. Subdirect decomposition', Trans. Amer. Math. Soc. 156, 335-342.

H. Lakser (1973), 'Principal congruences of pseudocomplemented distributive lattices', Proc. Amrel Math. Soc. 37, 32-36.

K. B. Lee (1970), 'Equational classes of distributive pseudocomplemented lattices', Canad. $J$. Math. 22 881-891.

E. Marczewski (1958), 'A general scheme of the notions of independence in mathematics', Bull. Acad. Polon. Sci. 6 731-736.

A. Urquhart (1973), 'Free distributive pseudo-complemented lattices', Algebra Universalis 3, 13-15.

University of Illinois at Chicago

Chicago, Illinois

U. S. A. 